\title{
Functional characterization of the conservative protein CG17337 in Drosophila melanogaster
}

\author{
E. Andreyeva ${ }^{1 *}$, A. Ogienko ${ }^{1}$, E. Kozhevnikova ${ }^{1,2}$, A. Ivankin ${ }^{1}$, L. Yarinich ${ }^{1}$, \\ G. Pavlova ${ }^{1}$, J. Popova ${ }^{1,2}$, A. Alekseeva ${ }^{1}$, T. Dubatolova ${ }^{1}$, S. Kopyl ${ }^{1}$, A. Pindyurin ${ }^{1}$ \\ ${ }^{1}$ Institute of Molecular and Cellular Biology SB RAS, Novosibirsk, Russia \\ ${ }^{2}$ Institute of Cytology and Genetics SB RAS, Novosibirsk, Russia \\ *e-mail: andreeva@mcb.nsc.ru
}

Key words: metallopeptidase M20, CG17337, CNDP2, cell cycle, cell death, cancer, lifespan, senescence

Motivation and Aim: Eukaryotic peptidases play an important role in protein and peptide metabolism. Currently, close attention is being paid to the human CNDP2 gene encoding a protein of metallopeptidase M20 family. The CNDP2 is a tumor suppressor protein; changes of its expression level stimulate cell proliferation and are used as a biomarker of cancer. Our aim was to clarify the function of drosophila CG17337 gene, which is the ortholog of $C N D P 2$, at the level of cells and whole organism.

Methods and Algorithms: First, we have raised polyclonal antibodies specific to CG17337, which allowed us to visualize this protein in drosophila tissues and cultured cells by confocal microscopy. Second, using CRISPR/Cas9-mediated homologous recombination, we generated null mutant of the CG17337 gene. The CG17337 nullmutation was checked by genotyping PCR, DNA sequencing and Western Blot analysis. Also, we generated transgenic flies carrying genomic copy of the CG17337 gene for rescue experiments. We estimated influence of the CG17337 null-mutation on cell cycle in different tissues as well as on the lifespan of drosophila.

Results: We provide first insights on the role of the CG17337 protein in drosophila. First, we found that CG17337 is not only extracellular protein as was reported earlier [1], but also plays a role both in the cytoplasm and nucleus (chromatin). Particularly, we found CG17337 in a co-immunoprecipitation assay as a putative interactor partner of the SUUR (Suppressor of Underreplication) protein in embryonic nuclear extracts. The nuclear localization and chromatin association of CG17337 was observed in cell cycledependent manner. We determined by Fly-FUCCI approach that the CG17337 protein is enriched in G2 cells. Second, we have shown that depletion/lack of CG17337 in cultured S2 cells or in drosophila tissues leads to changes in mitotic cell cycle, whereas endocycle is not affected. Finally, the CG17337 is a dominant and haploinsufficient gene. The obtained null-mutation appeared to be homozygous viable and is characterized by an elongated female lifespan. Unexpectedly we found that females null mutant for the CG17337 gene display a prolonged period of fertility probably due to suppression of cell death in middle oogenesis during senescence.

Conclusion: Altogether, our results indicate that the drosophila CG17337 protein is necessary for proper mitotic cell cycle progression and cell death control.

Acknowledgements: The work was supported by RFBR grant No. 16-04-01598.

References

1. Karlsson et al. (2004). Proteomic analysis of the Drosophila larval hemolymph clot. J. Biol. Chem. 279(50):52033-52041. 